International Journal of Modern Physics A,

(c) World Scientific Publishing Company

\title{
DYNAMICAL GENERATION OF SOLITONS IN A $1+1$ DIMENSIONAL CHIRAL FIELD THEORY: NON-PERTURBATIVE DIRAC OPERATOR RESOLVENT ANALYSIS *
}

\author{
JOSHUA FEINBERG \& A. ZEE \\ Institute for Theoretical Physics, \\ University of California, Santa Barbara, CA 93106, USA
}

\begin{abstract}
We analyze the $1+1$ dimensional Nambu-Jona-Lasinio model non-perturbatively. We study non-trivial saddle points of the effective action in which the composite fields $\sigma(x)=\langle\bar{\psi} \psi\rangle$ and $\pi(x)=\left\langle\bar{\psi} i \gamma_{5} \psi\right\rangle$ form static space dependent configurations. These configurations may be viewed as one dimensional chiral bags that trap the original fermions ("quarks") into stable extended entities ("hadrons"). We provide explicit expressions for the profiles of some of these objects and calculate their masses. Our analysis of these saddle points, and in particular, the proof that the $\sigma(x), \pi(x)$ condensations must give rise to a reflectionless Dirac operator, appear to us simpler and more direct than the calculations previously done by Shei, using the inverse scattering method following Dashen, Hasslacher, and Neveu.
\end{abstract}

\section{Introduction}

In this talk we describe a novel method 3 for studying the non-perturbative spectrum of the $1+1$ dimensional Nambu-Jona-Lasinio (NJL) mode11

$$
S=\int d^{2} x\left\{\sum_{a=1}^{N} \bar{\psi}_{a} i \not \partial \psi_{a}+\frac{g^{2}}{2}\left[\left(\sum_{a=1}^{N} \bar{\psi}_{a} \psi_{a}\right)^{2}-\left(\sum_{a=1}^{N} \bar{\psi}_{a} \gamma_{5} \psi_{a}\right)^{2}\right]\right\} .
$$

The action (1.1) describes $N$ self interacting massless Dirac fermions $\psi_{a}(a=$ $1, \ldots, N$ ), and we study it in the limit $N \rightarrow \infty$ holding $N g^{2}$ finite (the large $N$ limit). This model is interesting because it shares with QCD some of its important

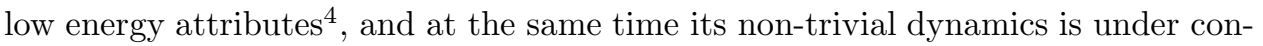
trol. Indeed, (1.1) is invariant under $S U(N)_{f} \otimes U(1) \otimes U(1)_{A}$. It is asymptotically free and exhibits dynamical mass generation (for $N \geq 2$ ) due to its infra-red instabilities. These instabilities may also polarize the vacuum inhomogenously, giving rise to chiral solitons 3 that are reminiscent of chiral bags 6.0 which model hadron formation. These chiral solitons and the small fermionic fluctuations around them, are the main subject of this talk. We rewrite (1.1) as

$$
S=\int d^{2} x\left\{\bar{\psi}\left[i \not \partial-\left(\sigma+i \pi \gamma_{5}\right)\right] \psi-\frac{1}{2 g^{2}}\left(\sigma^{2}+\pi^{2}\right)\right\}
$$

*Talk delivered by J.Feinberg at the Workshop on Low Dimensional Field Theory at Telluride, CO (August 1996). To appear in the conference proceedings. 
Dynamical Generation of Solitons...

where $\sigma(x), \pi(x)$ are the scalar and pseudoscalar auxiliary fields, respectively, ${ }^{\dagger}$ which are both of mass dimension 1 . These fields are singlets under $S U(N)_{f} \otimes U(1)$, but transform as a vector under the axial transformation, namely $\sigma+i \gamma_{5} \pi \rightarrow$ $e^{-2 i \gamma_{5} \beta}\left(\sigma+i \gamma_{5} \pi\right)$. Gaussian integration over the fermions in $(1.2)$ leads to the partition function $\mathcal{Z}=\int \mathcal{D} \sigma \mathcal{D} \pi \exp \left\{i S_{\text {eff }}[\sigma, \pi]\right\}$ where the bare effective action is

$$
S_{e f f}[\sigma, \pi]=-\frac{1}{2 g^{2}} \int d^{2} x\left(\sigma^{2}+\pi^{2}\right)-i N \operatorname{Tr} \ln \left[i \not \partial-\left(\sigma+i \pi \gamma_{5}\right)\right]
$$

and the trace is taken over both functional and Dirac indices.

The non-perturbative vacuum of (1.3) is determined, in the large $N$ limit 3 , by the simplest large $N$ saddle points of (1.3) where $\sigma$ and $\pi$ develop spacetime independent expectation values. These saddle points are extrema of the effective potential $V_{\text {eff }}$, which as a result of chiral symmetry depends only on the combination $\rho^{2}=\sigma^{2}+\pi^{2}$. It is minimized at $\rho=m \Lambda e^{-\frac{\pi}{N g^{2}(\Lambda)}}$, where $m$ is the dynamical mass of fermions. This mass is determined by the (bare) gap equation 3

$$
-m+i N g^{2} \operatorname{tr} \int \frac{d^{2} k}{(2 \pi)^{2}} \frac{1}{\not k-m}=0,
$$

were $\Lambda$ is an ultraviolet cutoff. The mass $m$ must be a renormalization group invariant. Thus, the model is asymptotically free. The vacuum manifold of (1.2) is therefore a circle $\rho=m$ in the $\sigma, \pi$ plane, and the equivalent vacua are parametrized by the chiral angle $\theta=\arctan \frac{\pi}{\sigma}$. Therefore, small fluctuations of the Dirac fields around the vacuum manifold develop dynamical ${ }^{\ddagger}$ chiral mass $m \exp \left(i \theta \gamma_{5}\right) . \S$

Static, space dependent solutions of the saddle point equations of (1.3)

$$
\begin{aligned}
\frac{\delta S_{\text {eff }}}{\delta \sigma(x, t)} & =-\frac{\sigma(x, t)}{g^{2}}+i N \operatorname{tr}\left[\left\langle x, t\left|\frac{1}{i \not \partial-\left(\sigma+i \pi \gamma_{5}\right)}\right| x, t\right\rangle\right]=0 \\
\frac{\delta S_{\text {eff }}}{\delta \pi(x, t)} & =-\frac{\pi(x, t)}{g^{2}}-N \operatorname{tr}\left[\gamma_{5}\left\langle x, t\left|\frac{1}{i \not \partial-\left(\sigma+i \pi \gamma_{5}\right)}\right| x, t\right\rangle\right]=0
\end{aligned}
$$

are the chiral solitons (at rest) mentioned above. The NJL model, with its continuous symmetry, does not have topologically stable soliton solutions. The solitons arising in the N.JL model can only be stabilized by binding fermions and releasing binding energy 10 . This observation is clarified further by comparing the NJL model to the Gross-Neveul (GN) model, namely, (1.1) with the $\gamma_{5}$ term deleted. The GN model possesses only a discrete symmetry, $\psi \rightarrow \gamma_{5} \psi$, rather than the continuous symmetry of the NJL model. This discrete symmetry is dynamically broken by the doubly degenerate non-perturbative vacuum, and thus there is a topologicaly

\footnotetext{
${ }^{\dagger}$ From this point on flavor indices are suppressed.

${ }^{\ddagger}$ Note that the axial $U(1)$ symmetry protects the fermions from developing a mass term to any order in perturbation theory.

$\S$ Note in pgssing that the massless fluctuations of $\theta$ along the vacuum manifold decouple from the spectrimn 8 so that the axial $U(1)$ symmetry does not break dynamically in this two dimensional model 9 .
} 
stable kink solution 1122.13, the so-called Callan-Coleman-Gross-Zee (CCGZ) kink, that interpolates between the two vacua. The kink binds any number $n \leq N$ of fermions in its single zero energy bound state, without affecting its mass. Its stability is guaranteed by topology already. In contrast, the stability of the extended objects arising in the NJL model is not due to topology, but to dynamics.

Shei 1 has found certain static solutions of (1.5) by applying the inverse scattering method 14 , following a similaranalysis by Dashen et al12. Recently, one of us developed an alternative method 13, 15 , based on the Gel'fand-Dikii identity 16 , to investigate spectra of such low dimensional field theories. We feel that this method has certain advantages over the inverse scattering method for analysing this type of problems, as it bypasses some of its technical steps. This talk is based on a paper 3 where we have applied this new method to study (1.1). In particular, our proof that the Dirac operator in the background of the static solutions of (1.5) is reflectionless is extremely simple, and does not require invoking inverse scattering techniques. It is actually valid for finite $N$. It is worth mentioning at this point that the NJL model (1.1) is completely integrable for any number of flavors 17 and thus its exact spectrum and $S$ matrix are known in detail The large $N$ spectrum we describe组 is consistent with the relevant exact resuts in 1 . However, the powerful methods of 17 are inherently limited to $1+1$ dimensional integrable models, whereas the analysis we describe here is potentially applicable to $1+1$ dimensional non-integrable models as well as to analysing inhomogeneous symmetric field configurations in higher dimensions 18 . 
Dynamical Generation of Solitons ...

\section{Absence of Reflections in the Dirac Operator With Static Back- ground Fields}

As was explained in the introduction, we need a manageable form of the diagonal resolvent of the Dirac operator $D=i \not \partial-\left(\sigma(x)+i \pi(x) \gamma_{5}\right)$ in a given background of static field configurations $\sigma(x)$ and $\pi(x)$. The extremum condition on $S_{\text {eff }}$ relates this resolvent, which in principle is a complicated and generally unknown functional of $\sigma(x), \pi(x)$ and of their derivatives, to $\sigma(x)$ and $\pi(x)$ themselves. This complicated relation is the source of all difficulties that arise in any attempt to solve the model under consideration. It turns out, however, that basic field theoretic considerations, that are unrelated to the extremum condition, imply that $D$ must be reflectionless. This spectral property of $D$ sets rather powerful restrictions on the static background fields $\sigma(x)$ and $\pi(x)$ which are allowed dynamically. In the next section we show how this special property of $D$ allows us to write explicit expressions for the resolvent in some restrictive cases, that are interesting enough from a physical point of view. Inverting $D$ has nothing to do with the large $N$ approximation, and consequently our results in this section are valid for any value of $N$.

The overall energy contained in any relevant static $\sigma, \pi$ configuration must be finite. Therefore these fields must approach constant vacuum asymptotic values, namely, points on the circle $\sigma^{2}+\pi^{2} \underset{x \rightarrow \pm \infty}{\longrightarrow} m^{2}$, with vanishing derivatives. We use the axial $U(1)$ symmetry to fix the coordinates in the $\sigma, \pi$ plane such that $\sigma(-\infty)=m$ and $\pi(-\infty)=0$. Then $\sigma(\infty)=m \cos \theta$ and $\pi(\infty)=m \sin \theta$, where $\theta$ is the chiral alignment angle of the vacuum at $x=+\infty$ relative to the vacuum at $x=-\infty$. We use the Majorana representation $\gamma^{0}=\sigma_{2}, \gamma^{1}=i \sigma_{3}$ and $\gamma^{5}=-\gamma^{0} \gamma^{1}=\sigma_{1}$ for $\gamma$ matrices. In this representation $D$ becomes

$$
D=\left(\begin{array}{cc}
-\partial_{x}-\sigma & -i \omega-i \pi \\
i \omega-i \pi & \partial_{x}-\sigma
\end{array}\right)
$$

We invert 2.1) by solving

$$
\left(\begin{array}{cc}
-\partial_{x}-\sigma(x) & -i \omega-i \pi(x) \\
i \omega-i \pi(x) & \partial_{x}-\sigma(x)
\end{array}\right) \cdot\left(\begin{array}{cc}
a(x, y) & b(x, y) \\
c(x, y) & d(x, y)
\end{array}\right)=-i \mathbf{1} \delta(x-y)
$$

for the Green's function of (2.1) in a given background $\sigma(x), \pi(x)$. By dimensional analysis, we see that the quantities $a, b, c$ and $d$ are dimensionles. We analyzed (2.2) in 3 . The quantities $a(x, y), c(x, y)$ and $d(x, y)$ in $(2.2)$ may all be inferred from

$b(x, y)$, through symmetries of (2.1). The quantity $b(x, y)$ is the Green's function of the one dimensional Sturm-Liouville operator

$-\partial_{x}\left[\frac{\partial_{x} b(x, y)}{\omega+\pi(x)}\right]+\left[\sigma(x)^{2}+\pi(x)^{2}-\sigma^{\prime}(x)-\omega^{2}+\frac{\sigma(x) \pi^{\prime}(x)}{\omega+\pi(x)}\right] \frac{b(x, y)}{\omega+\pi(x)}=\delta(x-y)$. 
Analysis of the asymptotic behavior of (2.2) and (2.3) turned out to be quite valuable. The physical asymptotic boundary conditions imposed on $\sigma, \pi$ are such that the potential term in (2.3) asymptotically a constant, $m^{2}-\omega^{2}$. We therefore deduced in 3 that the asymptotic behavior of the diagonal resolvent of (2.1) is

$$
\begin{aligned}
\left\langle x\left|-i D^{-1}\right| x\right\rangle \underset{x \rightarrow \pm \infty}{\longrightarrow} \quad & \frac{1+R(k) e^{2 i k|x|}}{2 k}\left[i \gamma_{5} \pi(x)-\sigma(x)-\gamma^{0} \omega\right] \\
& +\frac{R(k) e^{2 i k|x|}}{2} \gamma^{1} \operatorname{sgn} x
\end{aligned}
$$

where $k=\sqrt{\omega^{2}-m^{2}}$ and $R(k)$ is the reflection coefficient of the Sturm-Liouville operator in (2.3). Consider now the expectation value of fermionic vector current ${ }^{e}$ operator $j^{\mu}$ in the static $\sigma(x), \pi(x)$ background

$$
\left\langle\sigma(x), \pi(x)\left|j^{\mu}\right| \sigma(x), \pi(x)\right\rangle=\int \frac{d \omega}{2 \pi} \operatorname{tr}\left[\gamma^{\mu}\left\langle x\left|-i D^{-1}\right| x\right\rangle\right] .
$$

We thus find from (2.4) that the asymptotic behavior of the current matrix elements is such that $\left\langle\sigma(x), \pi(x)\left|j^{0}\right| \sigma(x), \pi(x)\right\rangle \underset{x \rightarrow \pm \infty}{\longrightarrow} 0$, but

$$
\left\langle\sigma(x), \pi(x)\left|j^{1}\right| \sigma(x), \pi(x)\right\rangle \underset{x \rightarrow \pm \infty}{\longrightarrow} \int \frac{d \omega}{2 \pi} R(k) e^{2 i k|x|} \operatorname{sgn} x
$$

where we used the fact that $\int \frac{d \omega}{2 \pi} \frac{\omega}{k} f(k)=0$ because $k(\omega)$ is an even function of $\omega$. Thus, an arbitrary static background $\sigma(x), \pi(x)$ induces space dependent fermion currents that do not decay fast enough as $x \rightarrow \pm \infty$, unless $R(k) \equiv 0$. Clearly, we cannot have such currents in our static problem and we conclude that as far as the field theory (1.2) is concerned, the fields $\sigma(x), \pi(x)$ must be such that the SturmLiouville operators in (2.3) and therefore the Dirac operator (2.1) are reflectionless.

One may also write equations for $\langle\sigma(x), \pi(x)|\bar{\psi} \psi| \sigma(x), \pi(x)\rangle$ and $\left\langle\sigma(x), \pi(x)\left|i \bar{\psi} \gamma_{5} \psi\right| \sigma(x), \pi(x)\right\rangle$ that are analogous to (2.5). For reflectionless $\sigma, \pi$ backgrounds, these equations boil down at the saddle point (where the expectation value of the scalar density is $-\sigma(x) / g^{2}$, and that of the pseudoscalar density is equal to $\left.-\pi(x) / g^{2}\right)$ into

$$
\frac{1}{N g^{2}}=\int \frac{d \omega}{2 \pi} \frac{1}{\sqrt{\omega^{2}-m^{2}}} .
$$

which is simply the gap equation (1.4), and thus holds to begin with.

The absence of reflections in (2.1) emerges here from basic principles of field theory, namely, that an initially static $\sigma(x), \pi(x)$ will not become time dependnt due to backreaction. Absence of reflections is thys valid beyond the large $N$ saddle point condition from which it was deduced in 22 . For reflectionless backgrounds (2.4) simplifies to

$$
\left\langle x\left|-i D^{-1}\right| x\right\rangle \underset{x \rightarrow \pm \infty}{\longrightarrow} \frac{1}{2 \sqrt{\omega^{2}-m^{2}}}\left[i \gamma_{5} \pi(x)-\sigma(x)-\gamma^{0} \omega\right]
$$

${ }^{e}$ In the following it is enough to discuss only the vector current, because the axial current $j_{5}^{\mu}=$ $\epsilon^{\mu \nu} j_{\nu}$ 
This expression has cuts in the complex $\omega$ plane corresponding to scattering states of fermions of mass $m$. These cuts must obviously persist away from the asymptotic region, and we make use of this fact in the next section.

\section{The Diagonal Resolvent for a Fixed Number of Bound States}

The requirement that the static Dirac operator (2.1) be reflectionless is by itself quite restrictive, but in order to actually derive explicit expressions for the resolvent in terms of $\sigma(x), \pi(x)$ and their derivatives we supplement it by assuming in addition that $\sigma(x)$ and $\pi(x)$ are such that the spectrum of (2.1) contains a prescribed number of bound states. In the following we concentrate on the diagonal resolvent $B(x)=$ $b(x, x)$ of (2.3). Being the diagonal resolvent of (2.3), $B(x)$ satisfiest the Gel'fandDikii identity 16

$$
\begin{aligned}
& \partial_{x}\left\{\frac{1}{\omega+\pi(x)} \partial_{x}\left[\frac{\partial_{x} B(x)}{\omega+\pi(x)}\right]\right\} \\
- & \frac{4}{\omega+\pi(x)}\left\{\partial_{x}\left[\frac{B(x)}{\omega+\pi(x)}\right]\right\}\left[\sigma(x)^{2}+\pi(x)^{2}-\sigma^{\prime}(x)-\omega^{2}+\frac{\sigma(x) \pi^{\prime}(x)}{\omega+\pi(x)}\right] \\
- & \frac{2 B(x)}{[\omega+\pi(x)]^{2}} \partial_{x}\left[\sigma(x)^{2}+\pi(x)^{2}-\sigma^{\prime}(x)+\frac{\sigma(x) \pi^{\prime}(x)}{\omega+\pi(x)}\right] \equiv 0 .
\end{aligned}
$$

If we were able to solve (3.1) for $B(x)$ in a closed form for any static configuration of $\sigma(x), \pi(x)$, we would then be able to express $\left\langle x\left|i D^{-1}\right| x\right\rangle$ in terms of the latter fields and their derivatives, and therefore to integrate (1.5) back to find an expression for the effective action (1.3) explicitly in terms of $\sigma(x)$ and $\pi(x)$. Invoking at that point Lorentz invariance of (1.3) we would then actually be able to write down the full effective action for space-time dependent $\sigma$ and $\pi$. Note moreover that in principle such a procedure would yield an exact expression for the effective action, regardless of what $N$ is. Unfortunately, deriving such an expression for $B(x)$ in general is a difficult task. It becomes manageable only for the special $\sigma, \pi$ backgrounds specified above.

We now sketch our treatment 3 of a reflectionless background that supports a single bound state. In such a case, $B(x, \omega)$ has to have a simple pole on the real $\omega$ axis, say at $\omega=\omega_{1}$, where clearly, $\left|\omega_{1}\right|<m$. There is also the continuum cut as in (2.8). The product of these two factors has dimension -2 in mass units. Any other singularity $B(x, \omega)$ may have in the complex $\omega$ plane must involve $x$ dependence as well, through the combination $\exp \left(i \sqrt{\omega^{2}-m^{2}} x\right)$. However, the requirement that the Dirac operator be reflectionless, rules this possibility out. The pole and cut then exhausts all allowed singularities of $B(x, \omega)$ in the complex $\omega$ plane. In addition, $B(x, \omega)$ is dimensionless, and therefore has to be of the form

$$
B(x, \omega)=\frac{B_{2}(x) \omega^{2}+B_{1}(x) \omega+B_{0}(x)}{\sqrt{m^{2}-\omega^{2}}\left(\omega-\omega_{1}\right)}
$$

where the dimension of the unknown function $B_{k}(x),(k=0,1,2)$ is $2-k$. These 
functions are combinations of $\sigma(x), \pi(x)$ and their derivatives. Substituting (3.2) into (3.1) results ${ }^{f}$ in a polynomial of degree six in $\omega$, with $x$ dependnet coefficients that has to vanish identically. In this way they form an over-determined set of seven differential equations in the five functions $B_{2}, \ldots, B_{0}, \sigma(x)$ and $\pi(x)$. However, this over-determined system has a solution 3 satisfying the boundary conditions (2.8),

$$
\begin{gathered}
B(x)=\frac{\omega+\pi(x)}{2 \sqrt{m^{2}-\omega^{2}}}+\frac{\sigma^{2}(x)+\pi^{2}(x)-\sigma^{\prime}(x)-m^{2}}{4\left(\omega-\omega_{1}\right) \sqrt{m^{2}-\omega^{2}}} \text { where } \\
\sigma(x)=m-\frac{m \sin \theta \tan \frac{\theta}{2}}{1+\exp \left[2 \omega_{1} \tan \frac{\theta}{2} \cdot\left(x-x_{0}\right)\right]} \text { and } \\
\pi(x)=-[\sigma(x)-m] \cot \frac{\theta}{2} .
\end{gathered}
$$

This linear relation between $\sigma$ and $\pi$ is not surprising, because $\sigma, \pi$ are the two components of an axial vector. Note that the boundary conditions at $x \rightarrow+\infty$ require

$$
\omega_{1} \tan \frac{\theta}{2}<0 .
$$

Our results (3.4) for $\sigma(x)$ and $\pi(x)$ agree with those of 6 . They have the profile of an extended object, a lump or a chiral "bag", of size of the order $\cot \frac{\theta}{2} / \omega_{1}$ centered around an arbitrary point $x_{0}$. Note that the profiles in (3.4) satisfy

$$
\rho^{2}(x)=\sigma^{2}(x)+\pi^{2}(x)=m^{2}-m^{2} \sin ^{2}(\theta / 2) \operatorname{sech}^{2}\left[\omega_{1} \tan \frac{\theta}{2} \cdot\left(x-x_{0}\right)\right] .
$$

Thus, as expected by construction, this configuration interpolates between two different vacua at $x= \pm \infty$. As $x$ increases from $-\infty$, the vacuum configuration becomes distorted. The distortion reaches its maximum at the location of the "bag", where $m^{2}-\rho^{2}\left(x_{0}\right)=m^{2} \sin ^{2}(\theta / 2)$ and then relaxes back into the other vacuum state at $x=\infty$. The arbitrariness of $x_{0}$ is, of course, a manifestation of translational invariance.

In 3 we also have partial results for the case of two bound states, at energies $\omega_{1}, \omega_{2}$. In particular we found that $y(x)=\sigma(x)-m$ satisfies the differential equation

$$
\begin{aligned}
& 2 \lambda\left(\omega_{1}+\omega_{2}\right)\left[4 m y^{2}+2\left(1+\lambda^{2}\right) y^{3}-y^{\prime \prime}\right] \\
& +\partial_{x}\left\{4\left(m^{2}+\omega_{1} \omega_{2}\right) y+6 m y^{2}+2\left(\lambda^{2}+1\right) y^{3}-y^{\prime \prime}\right\}=0
\end{aligned}
$$

where $\lambda=-\cot (\theta / 2)$, and $\pi(x)$ is given by (3.5). Note that if we set $\omega_{1}+\omega_{2}=0$ and $\pi(x)=0$ the spectrum becomes invariant under $\omega \rightarrow-\omega$, and we obtain the equation appropriate to the Gross-Neveu model.

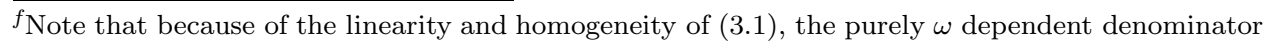
of (3.2) with its explicit dependence on the bound state energies factors out. 
Dynamical Generation of Solitons ...

\section{The Saddle Point Conditions}

Derivation of the explicit expressions of $\sigma(x)$ and $\pi(x)$ does not involve the saddle point equations (1.5). Rather, it tells us independently of the large $N$ approximation that $\sigma(x)$ and $\pi(x)$ must have the form given in (3.4) in order for the associated Dirac operator to be reflectionless and to have a single bound state at a prescribed energy $\omega_{1}$ in addition to scattering states. Thus, for the solution (3.4) we have yet to determine the values of $\omega_{1}$ and $\theta$ allowed by the saddle point condition (1.5). It is this dynamical feature that we can analyse only in the large $N$ limit. Note that the from (3.3) we can reconstruct the other three entries of $\left\langle x\left|-i D^{-1}\right| x\right\rangle$. Substituting this resolvent, with $\sigma, \pi$ given by (3.4) into (1.5) we obtain a quantization condition for $\omega_{1}$, in the form of coupled dispersion integrals that have to vanish. A nice feature of these integrals is that their potentialy ultra-violet divergent parts are equal to the difference of the two sides of the gap equation (2.7), and are therefore free of such divergences. Some $\omega$ poles in these integrals are at $x$ dependent locations. The quantization condition on $\omega_{1}$ cannot be $x$ dependent, so the residues of these poles must vanish. This happens provided the parameters in (3.4) satisfy

$$
\omega_{1}^{2}=m^{2} \cos ^{2}\left(\frac{\theta}{2}\right)
$$

This relation actually leaves $\theta$ the only free parameter in the problem with respect to which we have to extremize the action. The condition (3.6) then picks out one branch of (4.1). Assuming that the Dirac sea is completely filled, and that the bound state traps $n<N$ fermions, the saddle point condition becomes equivalent 3 to the requirement that $S_{\text {eff }}$ evaluated at the appropriate $\sigma, \pi$ configurations, be stationary as a function of $\theta$, namely,

$$
\frac{1}{N T} \frac{\partial S}{\partial \theta}=\frac{m}{2}\left(\frac{n}{N}+\frac{\theta}{2 \pi}\right) \sin \frac{\theta}{2} .
$$

Only the critical value at

$$
\theta=-\frac{2 \pi n}{N}
$$

corresponds to extended objects. The other zeros of $(4.2)$ at $\theta_{k}=2 \pi k, k \in \mathbf{Z}$ do not correspond to such objects at all as should be clear from (3.4). We therefore discard them, and concentrate on the extremum given by (4.3). The bound state energy is therefore $\omega_{1}=m \cos \left(\frac{n \pi}{N}\right)$. Equation (4.3) asserts that the relative chiral rotation of the vacua at $\pm \infty$ is proportional to the number of fermions trapped in the "bag". Our result (4.3) is consistent with the fact that the fermion number current in a soliton background can be determined in some cases by topological considerations 19 . Note from $(4.3)$, that in the large $N$ limit, $\theta$ (and therefore $\omega_{1}$ ) take on non-trivial values only when the number of the trapped fermions scales as a finite fracion of $N$.

As we already mentioned in the introduction, "bags" formed in the NJL model are not stable because of topology. They are stabilized by releasing binding energy 
of the fermions trapped in them. To see this more explicitly, we calculate now the mass of the "bag" corresponding to (3.4) and (4.3). Integrating (4.2) with respect to $\theta$ we find

$$
\frac{-1}{N T m} S(\theta)=\left(\frac{n}{N}+\frac{\theta}{2 \pi}\right) \cos \frac{\theta}{2}-\frac{1}{\pi} \sin \frac{\theta}{2} .
$$

Note that (4.4) is not manifestly periodic in $\theta$ because the Pauli exclusion principle limits $\theta$ to be between 0 and $2 \pi$. The mass of a "bag" containing $n$ fermions in a single bound state is given by the energy $E(\theta)=-S(\theta) / T$ evaluated at the appropriate chiral angle (4.3). We thus find that this mass is simply

$$
M_{n}=\frac{N m}{\pi} \sin \frac{\pi n}{N}
$$

in accordance with 5 . It is easy to check that (4.5) is a minimum of $E(\theta)$ for $0<\frac{n}{N}<1$. These "bags" are stable because

$$
\sin \frac{\pi\left(n_{1}+n_{2}\right)}{N}<\sin \frac{\pi n_{1}}{N}+\sin \frac{\pi n_{2}}{N}
$$

for $n_{1}+n_{2}$ less than $N$, such that a "bag" with $n_{1}+n_{2}$ fermions cannot decay into two "bags" each containing a lower number of fermions.

Entrapment of a small number of fermions cannot distort the homogeneous vacuum considerably, so we expect that $M_{n}$ will be roughly the mass of $n$ free massive fermions for $n<<N$ as (4.5) indeed shows. As a matter of fact we used this expectation to fix the integration constant in (4.4). However, as the number of fermions trapped in the "bag" approaches $N, M_{n}$ vanishes and the fermions release practically all their rest mass $N m$ as binding energy, to achieve maximum stability 10 . Note from (4.3), that the soliton twists all the way around as the number of fermions approaches $N$.

We conclude this talk making some remarks on the case of two bound states. In 3 we found that the bound state energies must be located at $\omega_{1}=m \cos \left(\frac{n_{1} \pi}{N}\right), \quad \omega_{2}=$ $m \cos \left(\frac{n_{2} \pi}{N}\right)$ which are identical in form to single bound state energy levels. From the general considerations of 19 we expect that the chiral angle $\theta$ will be proportional to the total number of fermions trapped by the "bag", so (4.3) must read now $\theta=-\frac{2 \pi\left(n_{1}+n_{2}\right)}{N}$. Clearly, the mass of the "bag" will depend on $n_{1}$ and $n_{2}$ seperately, and not only on their sum through $\theta$. 


\section{Acknowledgements}

JF would like to thank the organizers for an interesting and stimulating workshop. This work was partly supported by the National Science Foundation under Grant No. PHY89-04035.

\section{References}

1. Y. Nambu and G. Jona-Lasinio, Phys. Rev. 122, 345 (1961), ibid 124, 246 (1961).

2. D.J. Gross and A. Neveu, Phys. Rev. D 10, 3235 (1974).

3. J. Feinberg and A. Zee, Preprint NSF-ITP-96-15, March 1996 cond-mat/9603173).

4. For a recent review on the NJL model as an effective low energy description of QCD in four space time dimensions see J. Bijens, Phys. Rep. 265, 369 (1996).

See also R.T. Cahill and C.D. Roberts, Phys. Rev. D 32, 2419 (1985).

5. S. Shei, Phys. Rev. D 14, 535 (1976).

6. T. D. Lee and G. Wick, Phys. Rev. D 9, 2291 (1974); R. Friedberg, T.D. Lee and R. Sirlin, Phys. Rev. D 13, 2739 (1976); R. Friedberg and T.D. Lee, Phys. Rev. D 15, 1694 (1976), ibid. 16, 1096 (1977); A. Chodos, R. Jaffe, K. Johnson, C. Thorn, and V. Weisskopf, Phys. Rev. D 9, 3471 (1974).

7. W. A. Bardeen, M. S. Chanowitz, S. D. Drell, M. Weinstein and T. M. Yan, Phys. Rev. D 11, 1094 (1974); M. Creutz Phys. Rev. D 10, 1749 (1974).

8. See the concluding remarks in 5 who brings an unpublished argument for decoupling of $\theta$ due to R. Dashen, along the lines of M.B. Halpern, Phys. Rev. D 121684 (1975). See also E. Witten, Nucl. Phys. B145, 110 (1978).

9. S. Coleman, Commun. Math. Phys. 31, 259 (1973).

10. R. MacKenzie, F. Wilczek and A.Zee, Phys. Rev. Lett 53, 2203 (1984).

11. C.G. Callan, S. Coleman, D.J. Gross and A. Zee, unpublished; D.J. Gross in Methods in Field Theory, R. Balian and J. Zinn-Justin (Eds.), Les-Houches session XXVIII 1975 North Holland, Amsterdam, 1976); A. Klein, Phys. Rev. D 14, 558 (1976); See also 13 and R. Pausch, M. Thies and V. L. Dolman, Z. Phys. A 338, 441 (1991).

12. R.F. Dashen, B. Hasslacher and A. Neveu, Phys. Rev. D 12,2443 (1975).

13. J. Feinberg, Phys. Rev. D 51, 4503 (1995).

14. L.D. Faddeev and L.A. Takhtajan, Hamiltonian Methods in the Theory of Solitons (Springer Verlag, Berlin, 1987).

15. J. Feinberg, Nucl. Phys. B433, 625 (1995).

16. I.M. Gel'fand and L.A. Dikii, Russian Math. Surveys 30, 77 (1975).

17. N. Andrei and J.H. Lowenstein, Phys. Rev. Lett. 43, 1698 (1979), Phys. Lett. 90B, 106 (1980), ibid. 91B 401 (1980).

18. R.F. Dashen, B. Hasslacher and A. Neveu, Phys. Rev. D 10, 4130 (1974).

19. J. Goldstone and F. Wilczek, Phys. Rev. Lett. 47, 986 (1981); See also Eq. (4.44) of R. Aviv and A. Zee, Phys. Rev. D. 5, 2372 (1972). 